\title{
Extreme Events on Correlated Networks
}

\author{
Wang Fei $^{1}$, Fang Weiguo ${ }^{1}$ and Chen Chang $^{2}$ \\ ${ }^{1}$ School of Economics and Management Beihang University, Beijing 100191, China \\ ${ }^{2}$ Agricultural Bank of China, Beijing, China \\ wangfei@buaa.edu.cn
}

\begin{abstract}
In this paper, we study the extreme events taking place on complex net-works with different assortative net-work structures. Using random walk models, we build the formalism of extreme events on networks. We then present the definition of network as-sortativity, and discussed its relation-ship with the dynamical process of ex-treme events. It has been obtained that assortative networks leads to a high probability of extreme events and a low return interval. Moreover, it has been verified by simulation that decreasing the network assortativity is an effective method to prevent the ex-treme events.
\end{abstract} Networks

Index Terms - Extreme Events, Correlated Networks, Complex

\section{Introduction}

Extreme events, such as natural or technological disasters, challenge society's capabilities both for planning and response [1]. The response to extreme events offers numerous examples of how flexibility may contribute to the resilience of critical infrastructure systems. Traffic jams, web servers not responding, power black outs due to tripping of power grids are some of the common examples of extreme events. Specifically, the extreme events can be built on a complex network system. Above examples are built on transportation networks, web networks, power grids, respectively [2].

Without generality, such events can be thought of as an emergent phenomenon due to the transport on the net-works, i.e., numerous particles concentrate on a single node [3]. Therefore, extreme events can be defined as exceedences above a prescribed quantile.

However, previous literature about extreme events on complex networks mainly dealt with uncorrelated net-works, in which the degree correlation of the underlying networks is largely ignored [4]. Such approximation imposes limitations to revealing underlying network structures. Degree correlation refers to the likelihood that nodes with a given degree connect with nodes with similar degree. Most real networks do exhibit the presence of non-trivial cor-relations in their degree connectivity patterns. Empirical data show that high or low degree vertices of the network tend to preferentially connect to other vertices with similar degree, i.e., high degree nodes tend to connect with high degree nodes. In this situation, correlations are named assortative. In contrast, connections in many technological and biological networks are more likely to attach vertices of very different degree. Correlations are then referred to as disassortative. Assorative networks and disassortative networks are called correlated networks [5].

In this paper, we extend the research of extreme events on correlated networks to discuss the influence of assortative and dis assortative. We introduce the extreme events model in Sec.II in more detail. Then we simulate the model on correlated network in Sec.III and discuss the relative results. Finally, we conclude the paper in Sec.IV.

\section{Extreme Events Model}

In this section, we present extreme events model on complex network. We consider a fully connected, undirected, finite network with $\mathrm{N}$ nodes and $\mathrm{E}$ edges. We denote the network adjacency matrix by $A=\left(a_{i j}\right)_{N \times N}$, that is, $a_{i j}=1$ if node $i$ and node $j$ are connected and $a_{i j}=0$ otherwise.

On this network, we have $W$ walkers performing the standard random walk. These walkers are non-interacting. A random walker at time $t$ locating on $i$ th node with $k_{i}$ links can choose to hop to any of the neighboring nodes with the same probability. Thus, transition probability for going from $i$ th to $j$ th node is $a_{i j} / k_{i}$. Therefore, the master equation that elaborate the $n$-step transition probability of a walker starting from node $i$ at time $t$ to node $j$ at time $t+1$ is

$$
P_{i j}(t+1)=\sum_{n} \frac{a_{n j}}{k_{n}} P_{i n}(t) .
$$

The stationary state is

$$
\lim _{t \rightarrow \infty} P_{i j}(t)=\frac{k_{i}}{2 E} \stackrel{\text { def }}{=} p_{i} .
$$

Then, we deduce the distribution of random walkers $f_{k}(w)$ on a given node $i$. Obviously, it is binomial distribution, i.e.,

$$
f_{i}(w)=\left(\begin{array}{c}
w \\
W
\end{array}\right) p_{i}^{w}\left(1-p_{i}\right)^{1-w}
$$

Taken into account of the fluctuation, we can calculate the probability of finding more than $q$ walkers traverse a given node with degree $k$ by summing up the entire possible situations, that is ${ }^{[4]}$

$$
F(k)=\sum_{j=0}^{2 s} \frac{1}{2 s+1} \Phi
$$

where

$$
\Phi=\sum_{k=[q]+1}^{M}\left(\begin{array}{l}
M \\
k
\end{array}\right) p^{k}(1-p)^{M-k}
$$

and $M=W-s+j$.

By the definition, we designate an event to be extreme if the above equation is met. More than $q$ walkers gathered in a given node means that the congestion happened. In the next 
section, we will concentrate these parameter on different kinds of complex networks.

\section{Results and Simulation}

For clarity of later discussions, we introduce some common definitions about the correlated networks. The degree distribution is denoted by $p(k)$, which describe the probability that a randomly chosen node has degree $\mathrm{k}$. The edge end distribution, $q(k)$, is the probability that the degree of a node reached by a randomly selected edge is $k$. By definition, $q(k)=k p(k) /<k>$, where $\langle k\rangle=\sum k p(k)$ is the average degree.

The joint degree distribution $p(i, k)$ represents the probability that the two end nodes of a randomly selected edge have degree $i$ and $k$. Based on these parameters, we obtain the assortativity coefficient ${ }^{[5]}$

$$
r=\frac{1}{\sigma^{2}} \sum_{i, k} i k[p(i, k)-q(i) q(k)],
$$

where $\sigma^{2}=\sum_{k} k^{2} q(k)-\left(\sum_{k} q(k)\right)^{2}$ is the normalized coefficient. The assortativity coefficient describes the correlation of the entire complex networks. It can be found that $r>0$ corresponds to positive correlation while $r<0$ corresponds to negative correlation. Besides, $r \approx 0$ means the assortativity of the network is weak.

In the simulations, we use network with size $N=1000$. The results are verified by 100 different realizations. We mainly concern the relationship between the extreme events probability $F(k)$ and the network structure assortativity coefficient $r$.

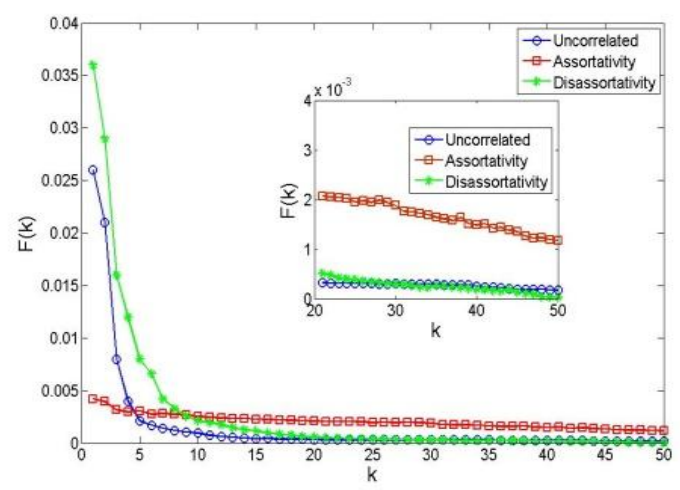

Fig. 1 The relationship between the extreme events occurrence probability and the degree.

First in Fig1, we investigate the relationship between the extreme events occurrence probability $F(k)$ and the degree $k$. From the figure we can see that the network structure plays an important role in the extreme events probability. In general, assortative networks tend to produce extreme events easily. For the lower degree nodes, extreme events happen more frequently on disassortative networks. In this type of network, high degree nodes prefer to connect with lower degree nodes, i.e., hub nodes transfer numerous walkers to low degree nodes, which make them easy to congest. On the other hand, for assortative networks, the flowability between hub nodes and low degree nodes are large, therefore extreme events probability has little difference for these two kinds of nodes.

Now we concern the return time $\tau$, which is defined as the interval between two extreme events in average. The results are shown in Fig 2.

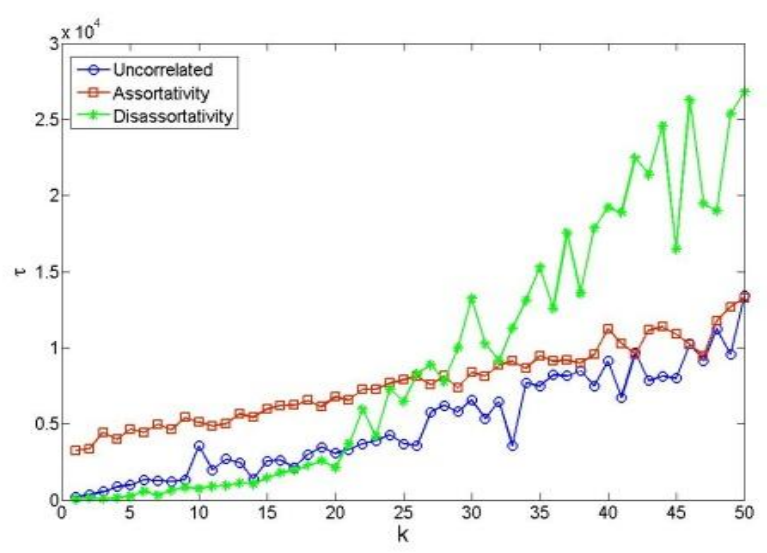

Fig. 2 The relationship between the return interval and the degree.

Compared with the curves in Fig 1, these results are logical. The curve of assortative network is relative smooth, while the curve of disassortative increases dramatically.

Finally, we present the relationship between the average return interval (averaged for all nodes) and the network structure assortativity coefficient $r$. We choose some representative results in Fig 3.

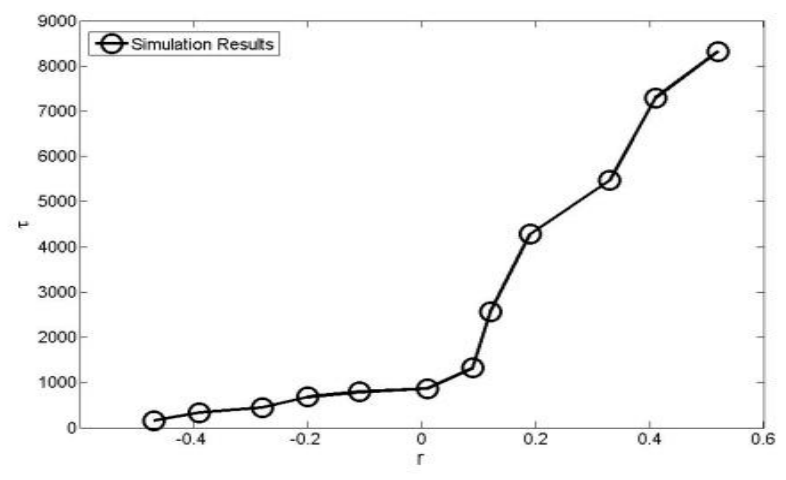

Fig. 3 The relationship between the average return interval and the assortativity coefficient..

In this figure, we can find that accompanied by the increase of assortativity coefficient, the extreme events probability shows an increment trend. This result tells us that to prevent the extreme events, one method is to decrease the assortativity of the networks.

\section{Conclusions}

To sum up, we investigated the ex-treme events phenomena in complex networks. We modeled this process 
and studied their dynamical results with the network assortativity struc-ture. We discussed the relative param-eters such as the occurrence probabil-ity and the return interval. It has been obtained that assortative networks leads to a high probability of extreme events and a low return interval. To prevent the extreme events, decline the network assortativity is an effective method.

\section{References}

[1] S. Albeverio, V. Jentsch and Holger Kantz (Ed.), Extreme events in nature and society, (Springer, 2005).

[2] J. D. Noh and H. Reiger, Phys. Rev. Lett. 92, 118701 (2004).

[3] R. Albert and A.-L. Baraba'si, Rev. Mod. Phys. 74, 47 (2002)

[4] V. Kishore, M.S. Santhanam, R.E. Amritkar.. Phys. Rev. Lett., 106, 188701 (2011)

[5] J. Zhou, G. Xiao, G. Chen. Phys. Rev. E, 88,032808 (2013). 